\title{
CARACTERÍSTICAS AMBIENTAIS DE CINCO IGARAPÉS DE TERRA-FIRME EM RESERVAS FLORESTAIS NO ESTADO DO AMAZONAS E SUA RELAÇÃO COM Batrachospermum cayennense (BATRACHOSPERMACEAE, RHODOPHYTA).
}

\author{
Domitila PASCOALOTO
}

Resumo - Foram investigados cinco igarapés de terra-firme com substrato arenoso localizados em área de reserva florestal nas proximidades da Rodovia BR-174 (Manaus - Boa Vista), Estado do Amazonas (02 $19^{\prime}$ $02^{\circ} 27^{\prime}$ S, $\left.59^{\circ} 45^{\prime}-60^{\circ} 05^{\prime} \mathrm{W}\right)$. Os igarapés foram visitados entre julho de 1996 e setembro de 1997 , e cada um foi percorrido em duas ocasiões, uma no período de menor precipitação ("seco") e outra no de maior precipitação ("chuvoso"). As variáveis ambientais analisadas foram: temperatura da água, concentração de oxigênio dissolvido, saturação de oxigênio, velocidade superficial da correnteza, cobertura do dossel, largura do igarapé, profundidade do igarapé, $\mathrm{pH}$, condutividade elétrica e turbidez. Espécimes de $B$. cayennense foram encontrados apenas no periodo "seco" em um dos igarapés e em ambos os periodos nos demais. Os resultados obtidos para os parâmetros fisicos e químicos foram, em linhas gerais, condizentes com aqueles registrados anteriormente para a região.

Palavras-chaves: igarapés de terra-firme, variáveis físicas e químicas, Batrachospermum cayennense, Floresta Amazônica, Brasil.

Environmental Characteristics of Five Terra-firme Streams from Forest Reserve Areas in Amazonas State and Their Relationship with Batrachospermum cayennense (Batrachospermaceae, Rhodophyta).

Abstract - Five sandy soil terra-firme streams in five forest reserves near BR-174 Road

(Manaus - Boa Vista) in Amazonas State $\left(02^{\circ} 19^{\prime}-02^{\prime} 27^{\prime} \mathrm{S}, 59^{\prime} 45^{\prime}-60^{\circ} 05^{\prime} \mathrm{W}\right)$ were investigated. The streams were visited from July/1996 to September/1997, and each one was searched through for two times, one in the "dry" period and other in the "rainy" one. The environmental variables analysed were: water temperature, dissolved oxygen concentration, dissolved oxygen saturation, current velocity, canopy cover, stream width, stream depth, $\mathrm{pH}$, specific conductance and turbidity. B. cayennense specimes were found just at the "dry" period in one of the streams, and in both of them in the others. The results for physics and chemical parameters were in agreement with that previosly recorded for this region.

Key-words: "terra-firme" streams, physical and chemical variables, Batrachospermum cayennense, Tropical Amazon Rainy Forest, Brazil.

\section{INTRODUÇÃO}

Vários fatores exercem influência sobre as características físicas e químicas das águas de rios e riachos. Para o estudo das características limnológicas de um ambiente, é necessário levar-se em conta sua origem (Maier, 1978). Na bacia Amazônica a litologia dos substratos e regime de erosão exercem controle fundamental na quimica das águas de superfície (Stallard \& Edmond, 1983). As águas que têm suas fontes na Amazônia Central refletem especialmente bem as condições extremas dessa região (Fittkau et al., 1975), apresentando, em sua maioria, uma pronunciada pobreza de sólidos inorgânicos dissolvidos, além de $\mathrm{pH}$ extremamente baixo (Sioli, 1951; Brinkmann \& Santos, 1973; Furch et al., 1982).

Comunidades vegetais bentônicas de

'INPA, CPGC, PDBFF/CPEC, C.P. 148 - 69011-970 - Manaus - AM, Brasil 
ambientes lóticos consistem de várias espécies, geralmente dominadas por algas e briófitas (Holmes \& Whitton, 1981). Entre as macroalgas, Batrachospermum (Batrachospermaceae, Rhodophyta) é um dos gêneros mais freqüentemente encontrados em igarapés localizados em áreas florestais (Sheath et al., 1989; Pascoaloto \& Necchi, 1990; Branco \& Necchi, 1996). Plantas de Batrachospermum spp. têm sido observadas na região de Manaus e arredores desde o século XIX (Dickie, 1881; Fittkau, 1964; Fittkau et. al., 1975; Uherkovich \& Franken, 1980; Necchi, 1990), entretanto, apenas recentemente (Pascoaloto, 1999) comunidades de espécies desse gênero, entre outras macroalgas, começaram a ser investigadas, sendo que a espécie mais freqüentemente encontrada foi Batrachospermum cayennense.

No presente estudo foram avaliadas algumas características físicas e químicas de 5 (cinco) igarapés de terra firme localizados em áreas de reserva florestal, onde encontravam-se presentes plantas de B. cayennense.

\section{MATERIAL E MÉTODOS Caracterização da Área Amostrada}

Os locais avaliados neste estudo estão localizados no município de Rio Preto da Eva (Figs. 1-2), com latitude $02^{\circ} 19^{\prime}-02^{\circ} 27^{\prime} \mathrm{S}$ e longitude $59^{\circ} 45^{\prime}$ $60^{\circ} 05^{\prime} \mathrm{W}$, região periférica norte segundo a divisão ecológica de Fittkau (1976). A região estudada pertence à Formação Alter do Chão (Período Terciário) (Schobbenhaus et al., 1984), e está incluída na Planície Amazônica, região do Baixo Platô Amazônico.
O estudo foi desenvolvido de julho/96 a setembro/97 em reservas do Projeto de Dinâmica Biológica de Fragmentos Florestais (PDBFF) do convênio INPA/SI (Instituto Nacional de Pesquisas da Amazônia/Smithsonian Institution). Foram avaliados igarapés de cinco reservas distribuídas em três fazendas (Dimona, Esteio e Porto Alegre), localizadas nas proximidades da rodovia BR-174 (Manaus - Boa Vista) (Figs. 1-2). As reservas estudadas foram Cabo Frio, Dimona, Florestal, Gavião e Porto Alegre (Fig. 2). Em cada uma foi amostrado um igarapé, preferencialmente o principal, denominados "Cabo Frio", "Dimona100", "Florestal", "Gaviãozinho", e "Porto Alegre", respectivamente. A escolha das reservas foi feita com base na presença de, no mínimo, um igarapé que percorresse sua extensão. Cada igarapé foi percorrido por toda sua extensão no interior da reserva em duas ocasiões, uma no período de maior precipitação (período "chuvoso", que costuma ocorrer entre os meses de dezembro e maio) e outra no de menor precipitação (periodo "seco", geralmente entre junho e novembro). Para cada trecho em que foram observadas plantas de B. cayennense, foi definida uma transeção permanente com 10 metros de extensão ao longo das margens (Holmes \& Whitton, 1981; Necchi \& Pascoaloto, 1993).

Os substratos dos igarapés estudados foram compostos por areia, raizes, troncos e galhos caídos, macrófitas, folhas e gravetos.

\section{Variáveis Biológicas}

As populações foram avaliadas 
segundo os parâmetros quantitativos freqüência e porcentagem de cobertura da espécie em cada ponto de amostragem.

A fim de se determinar a freqüência e porcentagem de cobertura a transeção, conforme definida anteriormente, foi dividida em 10 intervalos iguais. A freqüência foi determinada computando-se o número de intervalos em que cada espécie esteve presente. A porcentagem de cobertura foi estimada em cada intervalo e aproximada para toda a extensão utilizando-se $o$ seguinte indice: $1=$ $<1 \% ; 2=2-10 \% ; 3=11-20 \% ; 4=21-30 \%$; $5=31-40 \% ; 6=41-50 \% ; 7=51-60 \% ; 8=$ $61-70 \% ; 9=71-80 \% ; 10=81-100 \%$.

A porcentagem de cobertura

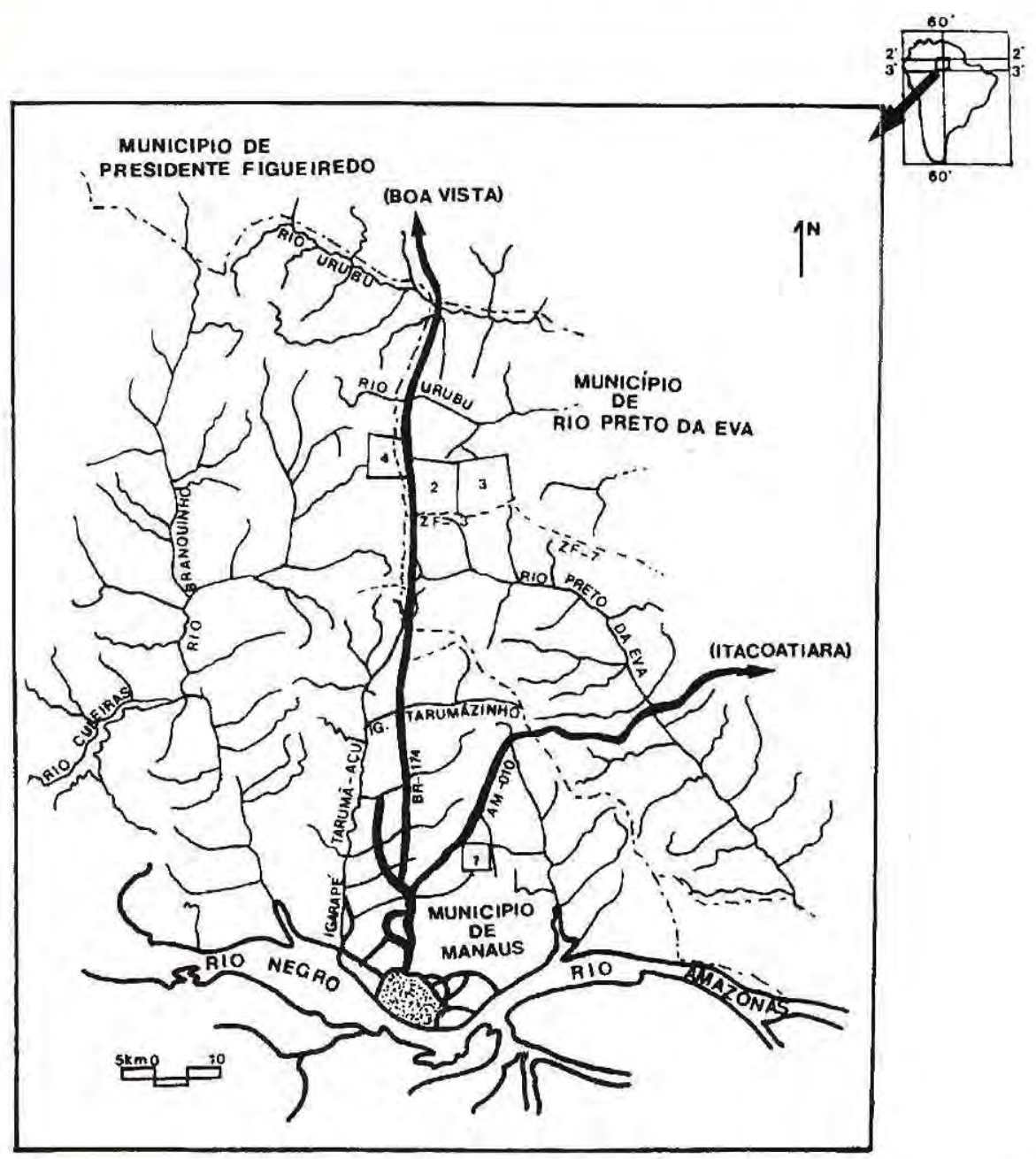

Fig.ura 1. Localização das áreas de estudo $(1=$ Fazenda Esteio, $2=$ Fazenda Porto Alegre, $3=$ Fazenda Dimona). 


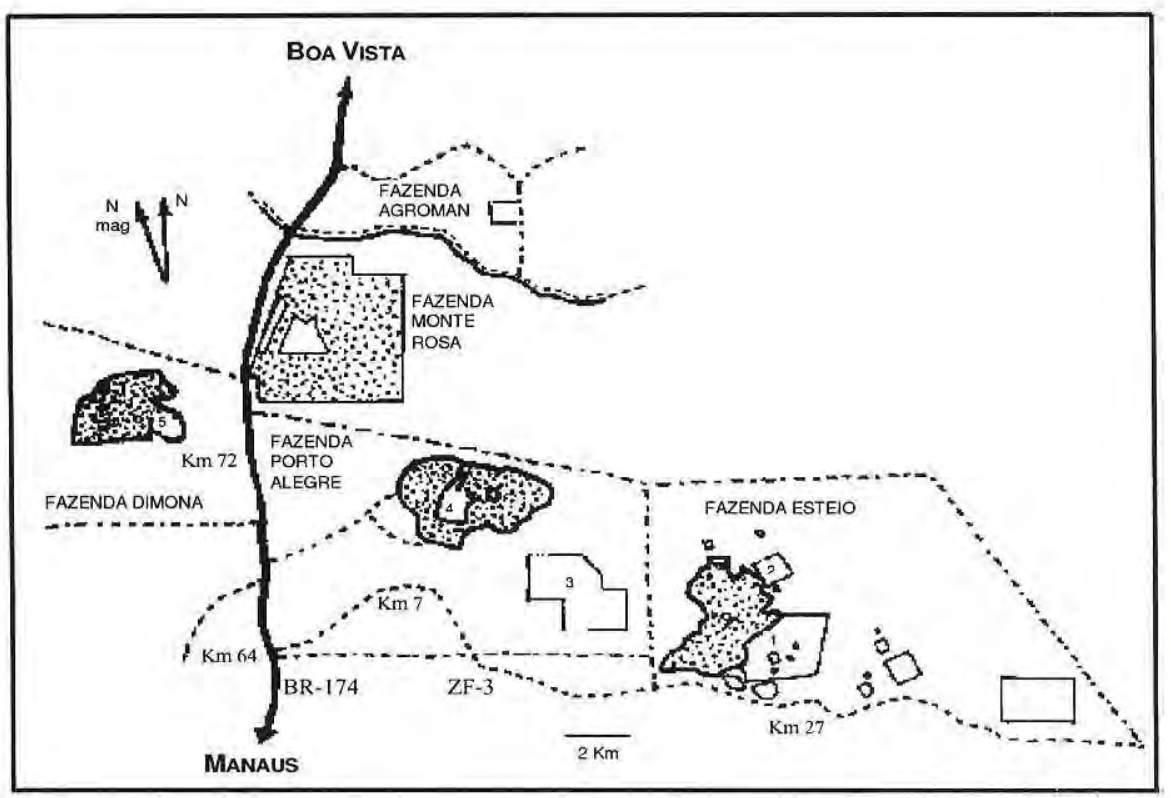

Figura 2. Localização das reservas estudadas $(1=$ Florestal, $2=$ Gavião, $3=$ Cabo Frio, $4=$ Porto Alegre, 5= Dimona).

fornece uma idéia da biomassa (Holmes \& Whitton, 1981). A escala utilizada foi baseada naquelas propostas em trabalhos anteriores sobre algas de ambientes lóticos, como Johanson (1982); Sheath \& Burkholder (1985); Necchi et al. (1991). As modificações na escala (maior número de intervalos, diminuindo a amplitude dentro deles) foram feitas tendo em vista o tipo de enfoque pretendido neste estudo e as características das áreas estudadas.

\section{Variáveis Ambientais}

Foram avaliadas as seguintes variáveis ambientais: temperatura da água, velocidade superficial da correnteza, cobertura do dossel, largura do igarapé, profundidade do igarapé, concentração de oxigênio dissolvido, saturação de oxigênio, $\mathrm{pH}$, condutividade e turbidez.

A temperatura foi medida com termômetro padrão de mercúrio, com graduação de 0 a $100^{\circ} \mathrm{C}$, no trecho médio da transeção. A velocidade superficial da correnteza foi estimada medindo-se o tempo gasto para que uma bóia de pesca de isopor percorresse a extensão da transeção (Sheath et al., 1986; Pascoaloto \& Necchi, 1990). Foram feitas 5 medições e calculada a média. A abertura do dossel foi avaliada através do densiômetro Lemmon Forest Densiometer Model-C, foram feitas duas medições, uma no início e outra no final da transeção. A determinação da largura e profundidade dos locais foi realizada utilizando-se uma trena com marcação em milímetro, centímetro e metro. Em ambos os 
parâmetros foram feitas 10 medições em pontos eqüidistantes na transeção, calculando-se as médias. As medidas de largura foram tomadas de margem a margem, enquanto que as de profundidade foram realizadas no trecho médio da correnteza.

A água para determinação do oxigênio foi coletada no trecho médio da transeção, sendo que a concentração de oxigênio dissolvido foi determinada segundo método de Winkler modificado (Golterman \& Clymo,1970). O cálculo da saturação de oxigênio foi feito com base na tabela de Golterman et al. (1978). A água para determinação dos demais parâmetros foi coletada no trecho médio da correnteza, na última estação amostrada naquela coleta, e as análises foram conduzidas no laboratório de química ambiental da Coordenadoria de Pesquisas em Geociências (CPGC) do INPA. O pH foi medido através de pHmetro digital WWR, cat. 34100-608. A condutividade elétrica foi avaliada com o uso de condutivímetro digital Jenway mod. 4010. A turbidez foi determinada com turbidimetro Polillab AP 1000II.

\section{Análise Estatística}

Foi realizado o teste de correlação (r) de Spearman (Freund \& Walpole, 1980) entre a freqüência da espécie e os parâmetros ambientais em cada segmento de cada igarapé, e entre o número de segmentos onde a alga foi observada ao longo do igarapé e o valor médio das variáveis físicas velocidade da correnteza, cobertura do dossel, largura e profundidade do igarapé. Foi utilizado um teste não paramétrico devido à natureza dos dados biológicos serem discretas, não fazendo sentido a utilização de um recurso matemático para torná-las contínuas, como o são os dados físicos e químicos (Costa Neto, 1977).

\section{RESULTADOS}

Foram encontrados, no total, 26 trechos de igarapés com a alga $B$. cayennense, sendo quatro (04) no igarapé Cabo Frio, um (01) no Dimona, um (01) no Florestal, dezenove (19) no Gaviãozinho e um (01) no Porto Alegre (Tabela 1). Aalga foi encontrada apenas no periodo de menor precipitação ("seco") no igarapé Porto Alegre, e em ambos os períodos nos demais. A grande extensão do igarapé Gaviãozinho não permitiu que todos os pontos fossem avaliados (variáveis biológicas e ambientais) nos dois períodos (embora visitas de inspeção revelassem que espécimes de $B$. cayennense estiveram presentes em todos os pontos em ambos os periodos).

Espécimes foram observados em um a sete intervalos, sendo os maiores valores de freqüência registrados nos igarapés Cabo Frio e Gavião.

Considerando-se o fato que cada ponto do igarapé Gaviãozinho só foi avaliado (variáveis ambientais e biológicas) uma vez, e que a população do igarapé Porto Alegre só foi observada em um dos periodos, na análise estatística cada ponto de cada igarapé só foi incluído com um registro (26 observações no total), evitando-se, assim, mascarar os resultados. Foi encontrada correlação positiva entre o número de trechos com presença da alga e a variável física profundidade do canal $(r=0,89, P<0,05)$. Não foi observada correlação $(\mathrm{P}<0,05)$ entre 
freqüência da espécie e os parâmetros físicos e químicos registrados em cada segmento de cada igarapé.

\section{DISCUSSÃO}

Os igarapés avaliados neste estudo apresentaram valores extremamente baixos de $\mathrm{pH}$, condutividade e turbidez, sugerindo águas quimicamente pobres (pouco mineralizadas) e foram, em linhas gerais, condizentes com aqueles registrados anteriormente para a região (Sioli, 1951, Brinkmann \& Santos, 1973; Furch et al., 1982; Campos, 1993). $\mathrm{O}$ valor baixo de $\mathrm{pH}$ encontrado nas águas estudadas favorece as plantas de
Batrachospermum, pois no $\mathrm{pH}$ encontrado $(<4,5)$ o carbono inorgânico disponivel encontra-se predominantemente como ácido carbônico $\left(\mathrm{H}_{2} \mathrm{CO}_{3}\right)$, forma utilizada pelas espécies de Batrachospermum, que inclusive não são capazes de utilizar bicarbonato $\left(\mathrm{HCO}_{3}^{-}\right)$, forma predominante de carbono inorgânico em $\mathrm{pH}$ mais elevado (Raven \& Beardall, 1981).

Os igarapés estudados apresentaram fluxos moderados $(12,0$ $\left.29,0 \mathrm{~cm} \cdot \mathrm{s}^{-1}\right)$ e foram bem oxigenados (saturação $>60 \%$ ), condições essas que favorecem o desenvolvimento de Rodófitas. Algas vermelhas de água doce podem ser encontradas em uma larga escala de concentração de oxigênio, variando de 0,2 a

Tabela 1. Valores obtidos (valor mínimo, máximo, com média e desvio padrão quando $n \geq 3$ ) para as variáveis ambientais nos igarapés estudados. ( $n=$ número de observações realizadas).

\begin{tabular}{|c|c|c|c|c|c|}
\hline Parâmetro/Local & $\begin{array}{l}\text { Cabo Frio } \\
(n=8)\end{array}$ & $\begin{array}{c}\text { Dimona } \\
(n=2)\end{array}$ & $\begin{array}{l}\text { Florestal } \\
(n=2)\end{array}$ & $\begin{array}{l}\text { Gavião } \\
(n=19)\end{array}$ & $\begin{array}{c}\text { Porto Alegre } \\
(n=1)\end{array}$ \\
\hline $\begin{array}{c}\text { Temperatura } \\
\left({ }^{\circ} \mathrm{C}\right)\end{array}$ & $\begin{array}{c}25,0-25,5 \\
(25,2 \pm 0,25)\end{array}$ & $24,0-25,0$ & $23,5-24,5$ & $\begin{array}{c}25,0-26,5 \\
(25,7 \pm 0,75)\end{array}$ & 24,5 \\
\hline $\begin{array}{c}\text { Velocidade da } \\
\text { correnteza }(\mathrm{cm} \cdot \mathrm{s}-1)\end{array}$ & $\begin{array}{c}21,87-24,27 \\
(23,07 \pm 1,20)\end{array}$ & $12,05-19,80$ & $21,28-25,00$ & $\begin{array}{c}23,07-27,36 \\
(25,21 \pm 2,14)\end{array}$ & 9,13 \\
\hline $\begin{array}{c}\text { Cobertura do } \\
\text { dossel }(\%)\end{array}$ & $\begin{array}{l}73,76-84,26 \\
(79,01 \pm 5,25)\end{array}$ & $74,26-85,18$ & $71,13-78,95$ & $\begin{array}{c}83,63-86,98 \\
(85,30 \pm 1,67)\end{array}$ & 67,25 \\
\hline $\begin{array}{l}\text { Largura do igarapé } \\
\qquad(\mathrm{m})\end{array}$ & $\begin{array}{c}2,19 \cdot 2,26 \\
(2,22 \pm 0,03)\end{array}$ & $2,31-2,50$ & $1,71-2,00$ & $\begin{array}{c}3,59-3,90 \\
(3,74 \pm 0,15)\end{array}$ & 1,92 \\
\hline $\begin{array}{l}\text { Profundidade do } \\
\text { igarapé }(\mathrm{cm})\end{array}$ & $\begin{array}{c}32,28-32,84 \\
(32,56 \pm 0,28)\end{array}$ & $8,71 \cdot 18,38$ & $25,31-28,40$ & $\begin{array}{c}27,36=49,80 \\
(38,58 \pm 11,22)\end{array}$ & 23,26 \\
\hline $\mathrm{pH}$ & $\begin{array}{c}4,1-4,2 \\
(4,1 \pm 0,05)\end{array}$ & $4,2-4,3$ & $4,0-4,2$ & $\begin{array}{c}4,3-4,4 \\
(4,3 \pm 0,05)\end{array}$ & 4,2 \\
\hline $\begin{array}{l}\text { Condutividade } \\
\text { (mS.cm-1) }\end{array}$ & $\begin{array}{c}7,80-8,90 \\
(8,35 \pm 0,55)\end{array}$ & $14,90-15,90$ & $8,90-10,20$ & $\begin{array}{c}5,65-12,60 \\
(9,12 \pm 3,47)\end{array}$ & 8,60 \\
\hline $\begin{array}{c}\text { Turbidez } \\
\text { (FTU) }\end{array}$ & $\cdots$ & $\cdots$ & $\ldots$ & $\begin{array}{c}3,40-4,20 \\
(3,80 \pm 0,04)\end{array}$ & 1,0 \\
\hline $\begin{array}{c}\text { Oxigènio } \\
\text { Dissolvido (mg.l-1) }\end{array}$ & $\begin{array}{c}5,52-6,68 \\
(6,10 \pm 0,58)\end{array}$ & $4,94-5,15$ & $5,90-7,15$ & $\begin{array}{c}7,05-7,44 \\
(7,24 \pm 0,19)\end{array}$ & 6,62 \\
\hline $\begin{array}{l}\text { Saturação de } \\
\text { oxigênio }(\%)\end{array}$ & $\begin{array}{c}66,83-80,87 \\
(73,85 \pm 7,02)\end{array}$ & $58,67-62,35$ & $84,17 \cdot 85,73$ & $\begin{array}{c}85,35-92,54 \\
(88,94 \pm 3,59)\end{array}$ & 78,54 \\
\hline Frequeência & $\begin{array}{c}3-7 \\
(4,75 \pm 1,7)\end{array}$ & 1 & 3 & $\begin{array}{c}1-6 \\
(2,32 \pm 1,45)\end{array}$ & $t$ \\
\hline $\begin{array}{l}\text { Indice de } \\
\text { Cobertura }\end{array}$ & 2 & 1 & 1 & 2 & 2 \\
\hline
\end{tabular}


21 mg.l-1 (Sheath \& Hambrook, 1990), entretanto existe um leve aumento na freqüência de ocorrência de Rhodophyta em concentrações de $\mathrm{O}_{2}$ mais elevadas (Sheath, 1984). Algumas espécies de Batrachospermum já foram reconhecidas como ocomentes apenas em locais torrenciais, ou, no mínimo, verificou-se que elas crescem melhor em águas que correm rapidamente, e por muitos anos acreditou-se que essas espécies de corredeiras cresceriam em riachos devido às temperaturas inferiores e águas com melhor aeração que neles ocorrem (Whittford, 1960). Existe evidência, contudo, que algumas espécies ocorrentes em locais com fluxos velozes têm uma taxa respiratória mais elevada do que as espécies lênticas correspondentes. Segundo Whittford (1960) a velocidade da correnteza deve exceder 15 $\mathrm{cm} . \mathrm{s}^{-1}$ para produzir efeitos típicos de água corrente, ou seja, a produção da camada limite entre o substrato e a água onde a velocidade é reduzida e ocorre um aumento na troca de material entre o organismo e o ambiente.

As algas podem apresentar diversos tipos de talo: emaranhados de filamentos, colônias gelatinosas, filamentos mucilaginosos, filamentos livres, pseudoparênquima, parênquima, tufos e incrustantes (Holmes \& Whitton, 1981; Sheath \& Cole, 1992), sendo que esses vários grupos morfológicos enfrentam a correnteza de diversas maneiras (Sheath \& Hambrook, 1988). Plantas de Batrachospermum cayennense, entre outras espécies desse gênero, encontram-se na coluna d'água e apresentam talo do tipo filamento mucilaginoso, o qual, essencialmente, cria uma fina camada gelatinosa fixa em torno do corpo da planta, reduzindo o fluxo direto.
A cobertura do dossel encontrada nos igarapés presentemente estudados foi elevada $(>60,0 \%)$, chegando a ser $>80,0 \%$ em alguns locais. Tal característica, além de ser um dos principais fatores responsáveis pela relativa estabilidade da temperatura da água observada ao longo do ano numa mesma estação (Pascoaloto, 1999), impede a entrada de luz, limitando a flora aquática e, consequentemente, a biota local em geral. A incidência luminosa é uma variável fundamental para algas bentônicas, permitindo a esses organismos fotossintetizar componentes inorgânicos em biomassa viva. Outros fatores ambientais tais como estabilidade do substrato, temperatura, nutrientes e predadores podem influenciar a distribuição e abundância dessas comunidades, mas intensidade luminosa adequada é claramente um pré-requisito para a existência fototrófica (Hill, 1996). Rodófitas de águas continentais exibem uma larga variedade de necessidade de intensidade de luz (Sheath, 1984), algumas espécies, por exemplo, ocorrendo apenas em locais densamente sombreados enquanto outras podem ser encontradas também em porções ensolaradas do corpo d'água. Todavia, devido aos seus aparelhos fotossintéticos, plantas de Rodófitas geralmente exigem intensidades luminosas inferiores àqueles de Clorófitas, as quais, aparentemente, estão mais adaptadas para utilizar altas irradiações (Steinman \& McIntire, 1987).

As algas foram encontradas principalmente em raizes e galhos caídos. 
Quedas de árvores e galhos são comuns no interior da floresta, e quando essa queda se dá com as árvores adjacentes ao igarapé, forma-se uma fonte de substrato em potencial para a fixação de plantas de Batrachospermum. Os igarapés estudados apresentaram largura reduzida (em geral $<2,5 \mathrm{~m}$ ). Esse fato, associado à queda de árvores resultante de processos naturais, geralmente decorrente de fenômenos atmosféricos (principalmente ventos e raios), favorece a formação de clareiras, onde além de haver substratos (troncos e galhos) em abundância, a cobertura oferecida pelo dossel das árvores sofre sensível redução, aumentando assim a radiação incidente. As condições encontradas nesses trechos favoreceriam a presença de plantas de Batrachospermum, conforme foi observado por Fittkau (1964), ainda que Pascoaloto (1999) tenha encontrado plantas de Batrachospermum em menos de $25 \%$ do total das clareiras observadas.

Os igarapés que apresentaram maior número de pontos com presença de $B$. cayennense (Cabo Frio e Gaviãozinho) apresentaram canais mais profundos, e foram dois dos três mais largos. Pascoaloto (1999) observou que outro fator aparentemente limitante na configuração encontrada foi o tipo de substrato disponivel para as algas, sendo que as plantas de $B$. cayennense foram predominantemente epifiticas. Ressalte-se que durante os treze meses em que a autora estudou a sazonalidade das espécies de macroalgas ocorrentes em 5 segmentos de igarapés, plantas de $B$. cayennense foram observadas em substrato rochoso apenas em duas ocasiões.

A biocenose nos igarapés da Floresta Úmida Amazônica foi formada não apenas pela temperatura constantemente alta e pouca luminosidade, mas também pelas condições eletroliticamente pobres (Fittkau, 1964). O gênero Batrachospermum apresenta características peculiares que lhe permite ser bem-sucedido em locais aparentemente inóspitos para o crescimento algal, podendo, inclusive, ser encontrado em qualquer época do ano. Essa é, provavelmente, a principal razão para que apenas macroalgas pertencentes a esse gênero tenham sido observadas anteriormente na região.

Populações de B. cayennense foram registradas anteriormente para a Malásia, Guiana Francesa e para os estados brasileiros do Amazonas, Mato Grosso e Sergipe (Necchi, 1990). Entretanto, pouco, ou quase nada, encontra-se registrado sobre 0 ambiente onde foram encontradas. No presente estudo verificou-se sua ocorrência em locais sombreados, com fluxos moderados, águas ácidas e bem oxigenadas. Pascoaloto (1999) observou que essa espécie foi a mais freqüente, presente em $90 \%$ dos igarapés de oito reservas florestais. Visitas aleatórias a igarapés de áreas não preservadas, próximas à região deste estudo, não revelaram a presença de plantas de $B$. cayennense, e nesses locais, inclusive, a espécie mais freqüente foi $B$. macrosporum (a qual não foi registrada nos igarapés de reservas florestais até o momento investigados).

\section{CONCLUSÃO}

As condições físicas e químicas observadas nos igarapés (sombreados, água com valores extremamente baixos de $\mathrm{pH}$, condutividade e turbidez, bem oxigenados, fluxos moderados) foram favoráveis à ocorrência da espécie. 
Os dois igarapés que apresentaram maior número de trechos com presença da espécie foram também os mais profundos. Nesses igarapés foram também observados os maiores valores de freqüência da espécie. Essa característica, associada à presença de substratos favoráveis (galhos e troncos), sugere que uma associação de fatores é necessária para que a espécie possa se estabelecer -e permanecer- no local. Entretanto, muitos podem ser os fatores responsáveis pela configuração observada, inclusive o simples acaso. Novos estudos a serem desenvolvidos em igarapés que nascem e percorrem o interior da floresta, bem como aqueles localizados em áreas desmatadas e/ou de acesso público, são, entretanto, necessários para melhor estabelecer-se as relações entre as comunidades de $B$. cayennense e o meio no qual elas se encontram.

\section{AGRADECIMENTOS}

Ao PDBFF pelo apoio logístico ao longo do projeto e autorização de acesso às reservas. Ao INPA pelo acesso à Reserva Ducke. À CPGC pelo auxilio e acesso aos seus equipamentos. À Antonia G.N. Pinto e M. Socorro Rocha pela leitura critica das versões preliminares deste manuscrito e valiosos comentários. À CAPES pela bolsa de doutorado. Ao Dr. Bruce Forsberg pelas sugestões e orientação da tese.

\section{Bibliografia citada}

Branco, C.C.Z, Necchi Jr, O. 1996. Distribution of stream macroalgae in the eastern Atlantic Rainforest of São Paulo State, southeastern Brazil. Hydrobiologia,
333:139-150.

Brinkamm, W.L.F., Santos, A. 1973. Natural waters in Amazonia. VI: Soluble calcium properties. Acta Amazonica, 3(2):33-40.

Campos, Z.E.S. 1993. Parâmetros fisicoquimicos em igarapés de água clara e preta ao longo da rodovia BR-174 entre Manaus e Presidente Figueiredo - AM. Manaus. Instituto Nacional de Pesquisas da Amazônia/Fundação Universidade do Amazonas, Dissertação de mestrado. 93p.

Costa Neto, P.L.O. 1977. Estatistica. Săo Paulo: Edgard Blücher.

Dickie, G. 1881. On algae from the Amazons and its tributaries. J. Limn. Soc., 18 (108): 123-132.

Fittkau, E.J. 1964. Remarks on limnology of Central-Amazon rain-forest stream. Verh. Internat. Verein. Limnol., 15:1092-1096. 1976. Esboço de uma divisão ecológica da Região Amazônica. In: Association pro Biologia Tropical, II Simposio y Foro de Biologia Tropical Amazônica,365-372.

Fittkau, E.J.; Junk, W.; Klinge, H.; Sioli, H. 1975. Substrate and vegetation in the Amazon region. In: Cramer, J. (ed.). Berichte der Internationalen Symposien der Internationalen Vereinigung für Vegetationskunde Herausgegeben von Reinhold Tüxen. Vaduz: J. Cramer, p.73-90.

Freund,J.E.; Walpole, R.E. 1980. Mathematical Statistics. 3. ed. New Jersey: PrenticeHall. 548 p.

Furch, K.; Junk, W.J.; Klinge, H. 1982. Unusual chemistry of natural waters from the Amazon region. Acta Cient. Venezoelana, 33:269-273.

Golterman, H.L.; Clymo, R.S. 1970. Methods for chemical analysis of fresh waters. Oxford: Blackwell Scientific Publication, 160p.

Golterman, H.L.; Clymo, R.S.; Ohsntad, M.A.M. 1978. Methods for chemical analysis of fresh waters. Boston: Blackwell, $214 \mathrm{p}$.

Haslan, S.M. 1978. River plants: the macrophytic vegetation of watercourses. Cambridge, Cambridge Univ., 396p.

Hill, W. 1996. Effects of light. In: Stevenson, 
R.J.; Bothwell, M.L.; Lowe, R.L. (eds.). Algal ecology: freshwater benthic ecosystems. London: Academic Press, Inc, p.121-149.

Holmes, N.H.T.; Whitton, B.A. 1981. Phytobenthos of River Tees and its tributaries. Freshwat. Biol., 11:139-163.

Johansson, C. 1982. Attached algal vegetation in running waters of Jämtland, Sweden. Acta Phytogeographyca Suecica, Uppsala, v. 71, p. $1-83$.

Maier, M.H. 1975. Considerações sobre caracteristicas limnológicas de ambientes lóticos. B. Inst. Pesca, 5(2):75-90.

Necchi Jr, O. 1990. Revision of the genus Batrachospermum Roth (Rhodophyta, Batrachospermales) in Brazil. Berlin: J. Cramer, 201p. (Biblioteca Phycologia, 84).

Necchi Jr, O.; Dip, M.R.; Goes, R.M. 1991. Macroalgae of a stream in southeastern Brazil: composition, seasonal variation and relation to physical and chemical variables. Hydrobiologia, Dordrecht, v. 213, p. 241-250.

Necchi Jr, O,; Pascoaloto, D. 1993. Seasonal dynamics of macroalgal communities in the Preto River basin, São Paulo, southeastern Brazil. Arch. Hydrobiol, 129(2):231-252.

Pascoaloto, D. 1999. Sazonalidade e distribuição de macroalgas em igarapés de terra firme em áreas de reserva florestal nas cercanias de Manaus, Estado do Amazonas. Instituto Nacional de Pesquisas da Amazônia/Fundação Universidade do Amazonas. Tese de doutorado. $231 \mathrm{p}$.

Pascoaloto, D.; Necchi Jr, O. 1990. Seasonal variation of Sirodotia delicatula Skuja (Rhodophyta, Batrachospermaceae) in a small stream from São Paulo State, southeastern Brazil. Rev. Brasil. Biol, 50(1):37-44.

Raven, J.A.; Beardall, J. 1981. Carbon dioxide as the exogenous inorganic source for Batrachospermum and Lemanea. Br. Phycol. J. London, 16(2):165-175.

Schobbenhaus, C.; Campos, D.A.; Derze, G.R.; Asmus, H.E. 1984. Geologia do Brasil.
Brasilia: DNPM, 501 p.

Sheath, R.G. 1984. The Biology of Freshwater Red Algae. In: Round, F.E.; Chapman, D.J. (eds.). Progress in phycological research. (v. 3), Bristol, Biopress, p. 89-157.

Sheath, R.G.; Burkholder, J.M. 1985. Characteristics of softwater streams in Rhode Island II: composition and seasonal dynamics of macroalgal communities. Hydrobiologia, Dordrecht, v. 128, p. 109-118.

Sheath, R.G.; Cole, K.M. 1992. Biogeography of stream macroalgae in the North America. J. Phycol., 28:448-460.

Sheath, R.G.; Hambrook, J.A. 1988. Mecanical adaptations to flow in freshwater red algae. J. Phycol., 24:107-111. 1990. Freshwater ecology. In: Cole, K.M.; Sheath, R.G. Biology of the Red Algae. Cambridge: Cambridge Univ., p.423-453.

Sheath, R.G.; Hamilton, P.B,; Hambrook, J.A.; Cole, K.M. 1989. Stream macroalgae of the eastern boreal forest region of North America. Can. J. Bot., 67:3353-3562.

Sheath, R.G.; Morison, M.O.; Korch, J.E.; Kaczmarczyk, D.; Cole, K.M. 1986. Distribution of stream macroalgae in south-central Alaska. Hydrobiologia, 135:259-269.

Sioli, H. 1951. Alguns resultados e problemas da limnologia amazônica. Boletim técnico do Instituto Agronómico do Norte, Belém, n. 24.

Stallard, R.F.; Edmond, J.M. 1983. Geochemistry of the Amazon. 2: The influence of geology and weathering environment on the dissolved load. Journal of Geophysical Research., 88: 9671-9688.

Steinman, A.D.; McIntire, C.D. 1987. Effects of irradiance on the community structure biomass of algal assemblages in laboratory streams. Can. J. Fish. Aquat. Sci., 44 (9): 1640-1648.

Uherkovich, V.B.; Franken, M. 1980. Aufwuchsalgen aus Zentralmazonischen Regenwaldbachen. Amazoniana. Kiel, 7:49-79.

Whitford, L.A. 1960. The current effect and growth of fresh-water algae. Trans. Am. Microsc. Soc., Lawrence, 79(3): 302-309.

\section{Aceito para publicação em 07/08/2001}

